\section{Causal association of gut microbiome on the risk of rheumatoid arthritis: a Mendelian randomisation study}

I read with interest the articles by Inamo ${ }^{1}$ and Alpizar-Rodriguez et $a l^{2}$ regarding the effects of the gut microbiome on the risk of rheumatoid arthritis (RA). The Mendelian randomisation (MR) study suggested that dysbiosis may be a secondary phenomenon, rather than a trigger, in the pathogenesis of RA, ${ }^{1}$ while the cohort study by Alpizar-Rodriguez et al suggested a role for intestinal dysbiosis in the development of RA. ${ }^{2}$ However, some methodological issues in the MR study must be discussed. First, I applied a two-sample MR analysis in the MR base platform to the same data analysed with the MR by Inamo. ${ }^{1}$ From this analysis, I could obtain 32 single nucleotide polymorphisms as instrumental variables. The MR estimates determined using inverse variance weighted (IVW) and MR-Egger regression analyses support a causal association between gut microbiome and the occurrence of RA (IVW: beta $=-0.024$, $\mathrm{SE}=0.007, \mathrm{p}=0.0006 ;$ MR-Egger: beta $=-0.027, \mathrm{SE}=0.009$, $\mathrm{p}=0.005$ ), while the weighted median approach yielded no evidence of a causal association between gut microbiome and RA (beta $=-0.005, \mathrm{SE}=0.003, \mathrm{p}=0.144$ ). Unlike the MR results by Inamo, ${ }^{1}$ a 'leave-one-out' analysis demonstrated that the IVW method without rs1230666 remained significant $(p=0.034)$ and no single single nucleotide polymorphism (SNP) was driving the IVW point estimate. Second, MR studies are susceptible to bias from pleiotropy. Therefore, sensitivity analysis is required to verify the validity of conclusions drawn from the MR study. ${ }^{3}$ Two methods are commonly used for sensitivity testing: a weighted median estimator, which provides valid estimates even if $50 \%$ of the SNPs are not valid instruments ${ }^{4}$ and MR-Egger regression, which tests for unbalanced pleiotropy and estimates the causal effect of an exposure on an outcome. ${ }^{5}$ Here, I found that the results of the MR analysis are supported by significant findings of the MR-Egger analysis (also similar to the IVW estimates), thus providing additional confidence in these findings. Considering that the weighted median estimator allowing $50 \%$ of the instruments to be invalid may be a conservative method and no method can provide an infallible test of causation, ${ }^{3}$ the MR data may provide support for previous observational studies that have shown an association between microbiome and RA. ${ }^{2}$ Thus, I believe that the findings of this MR study should be interpreted by taking the aforementioned methodological concerns into consideration. In conclusion, the MR analysis results may support epidemiological evidence for a relationship between gut microbiome and RA, ${ }^{2}$ suggesting further investigation on how much gut microbiome affects the development of RA.

\section{Young Ho Lee $\odot$}

Rheumatology, Korea University Medical Center, Seoul, Republic of Korea

Correspondence to Professor Young Ho Lee, Rheumatology, Korea University Medical Center, Seoul 02841, Republic of Korea; lyhcgh@korea.ac.kr

Competing interests None declared.

Patient consent for publication Not required.

Provenance and peer review Not commissioned; internally peer reviewed.

(c) Author(s) (or their employer(s)) 2020. No commercial re-use. See rights and permissions. Published by BMJ.

\section{Check for updates}

To cite Lee YH. Ann Rheum Dis Epub ahead of print: [please include Day Month Year]. doi:10.1136/annrheumdis-2019-216747

Received 1 December 2019

Accepted 5 December 2019

\section{SLinked}

http://dx.doi.org/10.1136/annrheumdis-2019-216767

Ann Rheum Dis 2020;0:1. doi:10.1136/annrheumdis-2019-216747

ORCID iD

Young Ho Lee http://orcid.org/0000-0003-4213-1909

\section{REFERENCES}

1 Inamo J. Non-causal association of gut microbiome on the risk of rheumatoid arthritis: a Mendelian randomisation study. Ann Rheum Dis 2019. doi:10.1136/ annrheumdis-2019-216565. [Epub ahead of print: 19 Nov 2019].

2 Alpizar-Rodriguez D, Lesker TR, Gronow A, et al. Prevotella copri in individuals at risk for rheumatoid arthritis. Ann Rheum Dis 2019:78:590-3.

3 Burgess S, Bowden J, Fall T, et al. Sensitivity analyses for robust causal inference from Mendelian randomization analyses with multiple genetic variants. Epidemiology 2017;28:30-42

4 Bowden J, Davey Smith G, Haycock PC, et al. Consistent estimation in Mendelian randomization with some invalid instruments using a weighted median estimator. Genet Epidemio/ 2016:40:304-14.

5 Bowden J, Davey Smith G, Burgess S. Mendelian randomization with invalid instruments: effect estimation and bias detection through Egger regression. Int J Epidemiol 2015;44:512-25. 